on each plant. Every row and column must contain all letters once, the remaining spaces being unoccupied. Further, every pair of rows (or columns) must be occupied simultaneously in the same number of columns (or rows). When the incomplete block solution is known, little difficulty has been found in completing the square. The non-existence of an incomplete-block solution is, however, a difficult matter to establish, and Dr. Youden indicated that nearly exhaustive trials so far suggested the nonexistence of the (arithmetically) possible $22 \times 22$ square having seven letters.

It was gratifying to observe that a large and predominantly mathematical audience showed the greatest interest in Dr. Youden's account of practical experimental requirements, and in the variety of applications which the known solutions open up in biological material.

In the short time available, Mr. Yates, whose work in this field is widely known, confined himself to explaining the logical genesis of the very beautiful Lattice square, commencing from the simple, triple or multiple lattice. This whole group of designs is adapted to the requirements of the plant breeder, who may need to test two hundred or more varieties in a single year. If the number of varieties is a perfect square, they may be cross-classified in a square lattice, of which the rows and columns supply the block contents in two contrasted types of replication. The block size is thus reduced to the square root of the number of varieties. In a triple lattice $\mathrm{a}$ third type of replication is supplied by choosing for the same block varieties having the same letter of a Latin square, which may always be found. Squares, the side of which is an odd number, or a multiple of 4 , will also always yield a fourth type of replication, making a quadruple lattice. For prime numbers, and, as appeared later in the discussion, for all powers of primes, a complete set of mutually orthogonal classifications is possible, leading, if one replication of each type is used, to one of the known solutions of the problem of randomized incomplete blocks. We have, however, free choice in the topographical arrangement of the plots, and if the number of varieties is the square of an odd number, such as 11, we may halve the number of complete replications by superimposing pairs of these in a Latin square, so that six $11 \times 11$ squares will suffice to give equal precision to all comparisons. This considerable advantage, combined with the high precision to be expected in Latin square designs, makes the scheme as attractive experimentally as it is mathematically elegant.

Mr. W. L. Stevens, of the Galton Laboratory, had a surprise in store, in the form of a demonstration of the fact that for any power of a prime a com. pletely orthogonal square exists. The converse had been asserted, on the basis of an erroneous proof by Wernicke in 1910, but the theorem had appeared to be probably true from the construction in 1936 of completely orthogonalized squares of sides 8 and 9 , by Yates and Fisher respectively. It was known to his associates that Mr. Stevens had already established a demonstration for the square of any prime number, but the very simple generalization which he gave had only occurred to him during the week.

Based on the theorem that if $s$ is a power of a prime, a field of $s$ symbols with the corresponding operations of addition, subtraction, multiplication and division can be defined so that the results of these operations fall within the field and are unique, we may define a Latin square by the equation

$$
u_{L}=u_{\lambda} u_{R}+u_{C} \quad u_{\lambda} \neq 0_{C}
$$

where $u_{R}, u_{C}$ and $u_{L}$ designate the row, column and letter of any element, and $u_{\lambda}$ is arbitrary apart from the restriction that it may not be zero.

Assigning its $s-1$ possible values to $u_{\lambda}$ generates $s-1$ Latin squares, and the uniqueness of the solution of the equations, regarded as simultaneous in $u_{R}$ and $u_{c}$

$$
\begin{aligned}
& u_{i}=u_{\lambda} u_{R}+u_{C}, \\
& u_{j}=u_{\mu} u_{R}+u_{C},
\end{aligned}
$$

shows that the element having a given letter $i$ in the first square, and a given letter $j$ in the second, is uniquely determined in row and column. Thus any two Latin squares of the set are orthogonal, and the whole constitutes a completely orthogonalized square.

It was interesting to learn that the $9 \times 9$ squares obtained in this way are different from one previously given by Yates, so that for the larger squares a multiplicity of completely orthogonal solutions is to be anticipated.

It is much to be regretted that the programme allowed no time for discussion. We should have liked to hear the reaction of many mathematicians in the audience, which remained to the end closely interested.

\section{R. A. Fisher.}

\title{
Plant Growth Substances
}

$\mathrm{F}_{\mathrm{t}}^{\mathrm{o}}$ OLLOWING a project first put forward in 1925 , the Committee on Intellectual Cooperation of the League of Nations and the International Council of Scientific Unions have agreed to collaborate in the calling of occasional conferences on well-defined fields. The first of these, on the plant growth substances, or phytohormones, was held in Paris on October 1-2, 1937, under the joint auspices of the C.I.C. and the International Union of Biological Societies.

The report of the conference,* which has just been

* Etudes et Recherches sur les Phytohormones: Première Réunion organisée en collaboration avec l'Union Internationale des Sciences Biologiques, Paris, 1 et 2 Octobre, 1937. Pp. xiv +125. (Paris : Institut International de Coopération Intellectuelle.) issued, contains eight contributed papers and discussion. Prof. Kögl (Utrecht) describes the determination of the chemical nature of auxins $a$ and $b$ and the isolation of biotin. Dr. Niels Nielsen (Copenhagen) discusses the substances promoting growth in the fungi, and the difficulties introduced by the varying abilities of organisms to synthesize different members of the group of active substances. The evidence associating the formation and action of auxins with oxidative metabolism is reviewed by Prof. Koningsberger (Utrecht), the phenomenon of bud inhibition and other correlations by Prof. Dostál (Brmo) and the relation between the phytohormones and plant tropisms by the chairman, Prof. Boysen 
Jensen (Copenhagen). Prof. G. S. Avery (New London, U.S.A.) makes a plea for the consideration of the auxins as protoplasm irritants or 'evoca. tors'. Prof. Bouillenne (Liège) discusses the action of auxins on cell division and root formation, and expresses the rather surprising opinion that although promotion of cambial division and inhibition of lateral bud development occur on applying pure auxins, at concentrations within the physiological range, to a variety of plants, nevertheless "under natural conditions" the auxins do not have these effects. Finally, Mlle. Zollikofer (Zurich) reviews the rather unsatisfactory state of the work on the effect of animal hormones on plant growth. It seems unfortunate that Prof. Nðmeč, who (according to the preface) proposed the conference, and who has long been active in the closely related field of regeneration, should not have been present.

As is natural in a field which is developing so rapidly, there are many matters of dispute, and most of the papers stress points of uncertainty rather than the well-established facts. The very extensive material presented, however, makes clear how impressive has been the progress in the last few years towards an understanding of the control of growth in plants. Perhaps the most interesting parts of the book are the discussions, which are reported in full. Naturally a conversation between half a dozen men who are really familiar with the field is of much greater value than the usual questions at a large meeting.

The fact that many substances, which are probably not present in plant tissues, have physiological effects almost indistinguishable from those of the naturally occurring auxins, raised much discussion. Animal hormones present parallel cases. The difficulty centred partly on nomenclature, and partly on the applicability of results obtained with these synthetic substances to the interpretation of natural processes. As to the former, an agreement was reached according to which two groups of growth substances are distinguished, $(a)$ the auxins, $(b)$ the bios group. The auxins comprise those actually present in plants (phytohormones proper), and those not present but exerting similar effects. The bios group includes aneurin, biotin, œstrin, etc., known to be present in plants, and a group of substances designated 'specific nutrients', such as mesoinositol, $\beta$-alanine, leucine, and pyruvic and gluconic acids. The grouping of these latter under bios, a term which up to now has had a rather specific meaning, seems wholly unjustified, both because 'specific nutrients' are difficult to define and because such a large and heterogeneous group of compounds is involved.

Another question raised was whether the action of auxins constitutes 'irritability' or not. However, this was soon found to be unprofitable for lack of a clear statement of the rather old-fashioned concept of 'irritability' or stimulus, and the related one of stimulus-substances (Reizstoffe).

At the close of the conference, Prof. Boysen Jensen invited the members to meet again in Copenhagen in September 1939. The success of this first venture would seem to justify further extension of these small conferences between specialists in definite fields of scientific activity.

KenNeth V. Thimann.

\section{Contributions of Engineering to Physics}

\section{$\mathrm{P}$} ROF. M. L. E. OLIPHANT, of the University of Birmingham, took as the subject of his evening discourse to the British Association, delivered at Cambridge on August 22, the contributions of engineers, more especially electrical engineers, to the science of physics. To cover the whole field in a single lecture would be impossible and so he confined himself to a few only of the technical contributions made by electrical engineers to physics. He began by making a quotation from a dinner speech of Lord Rutherford.

"For the greater part of my life I have been engaged in investigations to try to obtain a clearer idea of the relations between electricity and matter, and the all-important part that electrical charges play in the structure of the atoms of our material world. Yet, I would find it difficult to tell you what electricity is. It is so fundamental an entity in Nature that explanation is impossible. Yet our knowledge of the laws which electricity obeys is now so complete that we are able to predict with considerable confidence the performance of any piece of electrical machinery-provided, of course, it is large compared to the atom."

As Prof. Oliphant was a pupil of Lord Rutherford and naturally the inspiration which he gave to all who worked with him in his famous laboratory has coloured his outlook, he wisely contented himself by illustrating his lecture by examples taken from the Cavendish Laboratory and the work carried out there, going back to the time when sealing-wax and string were practically the only essentials of a piece of apparatus for physical research. Many physicists regret the passing of that direct simplicity of approach. They recognize that the physicist can no longer be self-sufficient. 'The time is therefore ripe for a review of the situation which may help us to see how the interactions of engineering and physics may best be employed to their mutual advancement.

Electrical engineering, by the provision of technical equipment and facilities and by nurturing physical research in its own laboratories, has made the greatest contribution to the advancement of physics. Prof. Oliphant pointed out that when Faraday in 1831 made fundamental experiments on the magnetic induction in an iron ring, he had to make his own insulated conductors by winding laboriously twine and wire together over layers of calico.

Probably the greatest contribution made byelectrical engineering to the physical sciences is the provision of an abundant and steady supply of electricity for laboratory purposes. Prof. Oliphant recalled the time when a small gas engine used to drive a dynamo, and this in turn was used to charge accumulators. The supply of electricity to a class was therefore limited, and the voltage drop made the experiments very difficult. Rutherford used to tell of the trouble of preparing each morning a number of Grove cells and how these used often to fail badly in the course of the day's work. In these days also, the only voltmeter in the Cavendish Laboratory was a Cardew hot-wire instrument which was very sluggish in its action and took a large current, so that in measuring the voltage of a small accumulator, great care had to be taken 